\title{
Inventory and Implications of Plant Use for Environmental Conservation in Visconde de Mauá, Serra da Mantiqueira, Brazil
}

\author{
Mariana Martins da Costa Quinteiro, Ana Mayumi Gonçalves \\ Tamashiro, Marcelo Guerra Santos, Luiz José Soares Pinto, \\ and Moemy Gomes de Moraes
}

\section{Research}

\begin{abstract}
The community of Visconde de Mauá is located in the Serra da Mantiqueira Environmental Protection Area, characterized by high mountain rainforest vegetation. Despite a resident population predominantly from outside the region, inhabitants follow local patterns of plant use. Local plant uses were identified using participant observation, semi-structured and informal interviews, and guided tours. Uses were sorted in categories: medicine, food, handicrafts, fuel, construction, ornamental, and symbolic. Among the categories, medicinal use included the largest number of plant species. Tourism is intense in the area and has already affected community patterns of plant use by reducing demand for food cultivation. Alternatively, tourism has instead spurned demand for fuel and handmade crafts, a practice deemed to be incompatible with the preservation of the environment and local plant species.
\end{abstract}

\section{Introduction}

The Atlantic Forest is a highly degraded tropical ecosystem (Viana \& Tabanez 1996) considered by some as the most endangered tropical forest in the world. The Atlantic Forest is included in a list of global biodiversity hotspots due to its high indices of endemism and biodiversity and threats from human activities (Myers et al. 2000). Ribeiro et al. (2009) estimated the existing Atlantic Forest cover ranges from $11.4 \%$ to $16 \%$ of its original size. This estimate, however, may be too optimistic; Sloan et al. (2014) estimated the natural intact vegetation of the Atlantic rainforest at $3.5 \%$.

Biodiversity conservation in the Atlantic Forest is severely threatened by soil erosion, loss of biological diversity, the invasion of foreign species, and the degradation of hydro- graphic basins (Pereira et al. 2006). Uncontrolled real estate expansion and tourism practices coupled with overexploration of endemic natural resources also contribute to forest destruction (Pavan-Fruehauf 2000).

Even so, southeastern Brazil still harbors the main preserved areas in the Atlantic Forest domain, and fragments of secondary forests constitute the majority of the remaining vegetation. Presently, only a few regions can be characterized as primary forests, and these are typically found in high altitude areas within Environmental Conservation Units (ECUs) (Câmara 2003).

However, for the effective protection of endangered ecosystems, additional measures are necessary. The development of management projects enlisting society as a responsible agent for environmental conservation is urgent

\section{Correspondence}

Mariana Martins da Costa Quinteiro, Universidade Federal Rural do Rio de Janeiro, Seropédica, Rio de Janeiro, BRASIL. marianaquinteiro@gmail.com

Ana Mayumi Gonçalves Tamashiro, Universidade Federal Fluminense, Niterói, Rio de Janeiro, BRASIL.

Marcelo Guerra Santos, Faculdade de Formação de Professores, Universidade do Estado do Rio de Janeiro, São Gonçalo, Rio de Janeiro, BRASIL.

Luiz José Soares Pinto, Faculdade de Formação de Professores, Universidade do Estado do Rio de Janeiro, São Gonçalo, Rio de Janeiro, BRASIL.

Moemy Gomes de Moraes, Departamento de Botânica, Instituto de Ciências Biológicas, Universidade Federal de Goiás, Goiânia, Goiás, BRASIL.

Ethnobotany Research \& Applications 14:027-047 (2015) 
(Silva \& Andrade 2005). In this respect, ethnobotanical studies may support the elaboration of appropriate practices for management of vegetation with utilitarian purposes; these practices employ time-tested traditional knowledge to solve community issues for conservation purposes (Albuquerque 1999).

Gathering information on the use of natural resources by traditional people has revealed models permitting their sustainable use (Albuquerque 1999). This is based on the knowledge of such groups about the use and protection of the biological resources (Arruda 1997). Therefore, studies in this perspective must be considered in environmental discussions.

In the Atlantic Forest biome, ethnobotanical research was carried out with artisanal fishers (Fonseca-Kruel \& Peixoto 2004, Hanazaki et al. 2000), local farmers (Medeiros et al. 2004, Pinto et al. 2006, Silva \& Andrade 2005), and in popular produce markets (Azevedo \& Silva 2006). Regarding these biome ecosystems, past studies have primarily focused on coastal plain formations (FonsecaKruel et al. 2006, Santos et al. 2009) and coastal forests (Hanazaki et al. 2000, Medeiros et al. 2004, Pinto et al. 2006). Mountainous biomes and the high mountain Atlantic Forest have yet to be studied using ethnobotanical methods.

Visconde de Mauá is a rural agglomeration of villages located in the larger Serra da Mantiqueira, a mountainous Atlantic Forest ecosystem in southeastern Brazil. The region has several environmental attractions, including, but not limited to, waterfalls, mountains, lakes, and preserved forest areas (Richter \& Souza 2013). Thus, tourism dominates economic activities in Visconde de Mauá (Neves \& Maia 2012, Richter \& Souza 2013) with both positive and negative impacts on the environment and on native communities. On a local scale, tourism can generate economic growth and population change as tourists later decide to retire or reside permanently in the area. However, tourism can promote deforestation and the unsustainable misuse of a landscape to meet its demands (Buckley 2012, Ruschmann 2013).

This work is a case study with the aim of generating an ethnobotanical inventory in the community of Visconde de Mauá, Brazil, relating the use of plant species to the tourism activities in this region and analyzing its consequences for the conservation of local ECUs.

\section{Methods}

\section{Study area}

Visconde de Mauá is located in Serra da Mantiqueira Environmental Protection Area on the periphery of Itatiaia National Park. For further reference, this area sits on the far western part of the state of Rio de Janeiro (RJ) while overlapping into the neighboring state of Minas Gerais. In total, it encompasses three municipalities: Resende, Itatiaia, and Bocaina de Minas. Visconde de Mauá comprises villages and valleys settled in the territory of Alto Rio Preto micro-basin, and in 2012 the estimated population was 8000 (Neves \& Maia 2012). The economic activities in Visconde de Mauá are based on tourism, motivated especially by the environmental characteristics of the region (Neves \& Maia 2012, Richter \& Souza 2013). In addition, gastronomy and craftwork trade help propel the local economy in response to the typical tourists' expressed interests.

Settled in the Atlantic Forest domain, the vegetation of Visconde de Mauá is a transition between mountain rainforest and high mountain rainforest (Oliveira-Filho et al. 2004). The climate is subtropical humid (Köppen's Cwa), marked by a dry winter and a hot and rainy summer with high humidity levels. The average annual temperature varies from $18^{\circ} \mathrm{C}\left(64^{\circ} \mathrm{F}\right)$ to $21^{\circ} \mathrm{C}\left(70^{\circ} \mathrm{F}\right)$ with an annual rainfall ranging $1500-1800 \mathrm{~mm}$ (59-71 inches). The region has ecological refuges with low forest relicts from the Pleistocene called high altitude fields (IBAMA 2007).

\section{Ethnobotanical survey}

The field work included monthly trips of ten days on average from January 2006 to October 2007. The collection sites used during the research were at 1024-1350 $\mathrm{m}$ (3360-4430 ft) in the rural communities of Mauá, Maromba, Maringá, Santa Clara, Lote 10, and Ponte dos Cachorros (Figure 1).

The research followed methodological recommendations adapted for field practices suggested by Albuquerque and Lucena (2004). The process was based on sociability, full participant observation, registering information in a field diary, and prior-approved recordings of formal interviews. Semi-structured and informal interviews were also performed to learn botanical knowledge.

The selection of interviewees focused on the local populous that ostensibly used plant resources, defined henceforth as generalists (Albuquerque \& Lucena 2004). Once the first generalist was identified, we used a snowball technique (Bailey 1994) to identify other generalists in the community who correspondingly helped identify other generalists. However, some of the interviewees were peer-recognized as more astute in their botanical knowledge, thus they were considered and designated as key informants. During the interviews, research personnel also collected information from key informants via freelisting. Guided tours and walk-in-the-woods were also used for direct verification of plants and in situ collection. The sampling scale was defined using a collectors' curve (accumulation curve), adapted for this research (Borba \& Macedo 2006). 


\section{Quinteiro et al. - Inventory and Implications of Plant Use for Environmental Conservation in Visconde de Mauá, Serra da Mantiqueira, Brazil}

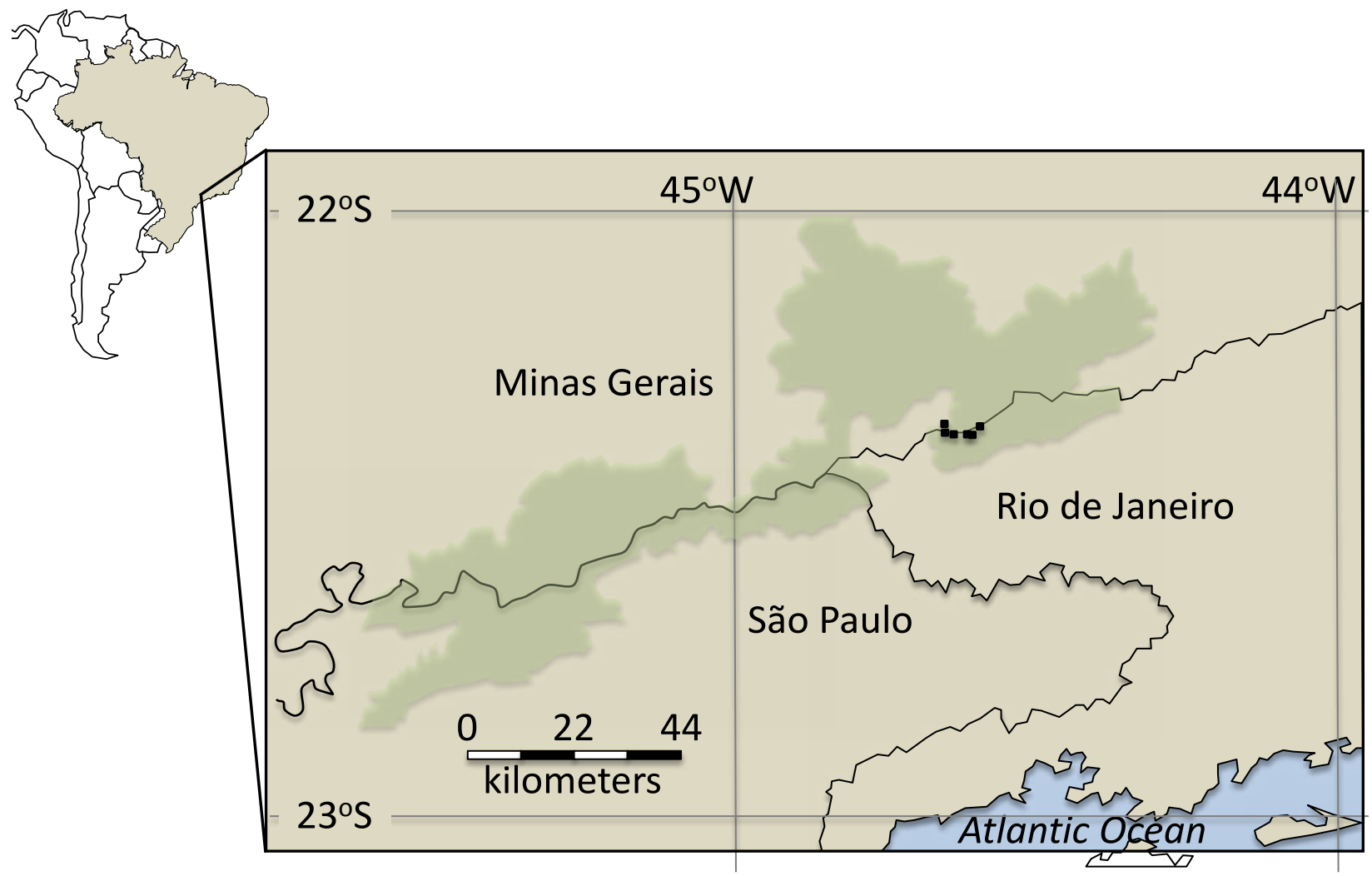

Figure 1. Study site and location of the Serra da Mantiqueira Environmental Protection Area (green), Rio de Janeiro, Brazil. Small squares denote the location of the villages of Visconde de Mauá: Mauá, Maromba, Maringá, Santa Clara, Lote 10, and Ponte dos Cachorros. Adapted from IBAMA (2007).

The plants were analyzed according to purpose and the plant part utilized, following the methods described in Borba and Macedo (2006) and Fonseca-Kruel and Peixoto (2004). The definition of categories of use of the plant resources was based on the considerations of Albuquerque and Andrade (2002), Fonseca-Kruel and Peixoto (2004), Shanley and Rosa (2005), and Vendruscolo and Mentz (2006) and are as follows:

1. Alimentation-plants eaten directly or used in preparation of products for household consumption;

2. Craftwork-plants used in manufacturing bijou, handicrafts, home ornaments, and other products intended for sale to obtain income;

3. Fuel wood-plants used for firewood such as leaves and branches used to ignite and sustain a fire;

4. Construction-plants used in building structures, fences, and furniture;

5. Cosmetic-plants used with aesthetic purposes;

6. Medicinal-plants used in prevention and treatment of specific diseases or indicated to help some organic function;

7. Ornamental-used in decoration of houses and gardens;

8. Symbolic-plants used in rituals, superstitions, and in the prevention and treatment of cultural diseases (e.g., quebrante, vento-virado, mau-olhado, descarrego).

Due to the importance of commerce for tourism in this community, a rank matrix was elaborated to verify the preferred species used in the craftwork category (Albuquerque \& Lucena 2004).

The collection of the botanical samples was performed under licensing from Brazilian Institute for the Environment - IBAMA (11307-1). The collected specimens were cataloged and identified using a specific bibliography and/ or via comparisons with exsiccates. The classification systems used were APG III for angiosperms (Reveal \& Chase 2011), Smith et al. (2006) for pteridophytes, and Kramer and Green (1990) for gymnosperms. The TROPICOS database (www.tropicos.org) and Lista de Espécies da Flora do Brasil (2014) were used to check nomenclatural information and also to verify whether the species was foreign or native. After cataloging, samples were deposited at the herbarium of the Universidade Federal do Rio de Janeiro (RFA). 


\section{Results}

\section{The respondents and their perceptions on plant resources}

Forty people were interviewed and categorized as 27 generalists and 13 key informants. These key informants were identified according to their activities in the following groups: general users, medicine collectors, faith-curers, healers, artisans, and small local farmers. When compared to the generalists, key informants constituted a distinct group (Table 1). The key informant group was almost equally comprised of men and women, while women predominantly made up the generalists group $(81.5 \%)$.

The majority of women in the key informant group (89\%) cultivated plants in their backyards. They reported the exchange of cultivated specimens with friends and neighbors, mainly for medicinal purposes; this practice occurred typically when their cultivated specimens were damaged or if they expressed interest in cultivating a previously unknown species of plant.

Table 1. Characteristics of all respondents and keyinformants from Visconde de Mauá, Rio de Janeiro, Brazil.

\begin{tabular}{|l|c|c|}
\hline Characteristic & All respondents & Key informants \\
\hline Male & $31 \%$ & $57 \%$ \\
\hline Female & $69 \%$ & $43 \%$ \\
\hline Age & $\begin{array}{c}38 \%>50 \\
\text { years-old }\end{array}$ & $\begin{array}{c}77 \%>50 \\
\text { years-old }\end{array}$ \\
\hline Education & $\begin{array}{c}31 \% \text { up to } \\
\text { elementary } \\
\text { school }\end{array}$ & $\begin{array}{c}62 \% \text { illiterate or } \\
\text { up to elementary } \\
\text { school }\end{array}$ \\
\hline Origin & $43 \%$ local & $85 \%$ local \\
\hline
\end{tabular}

Contrasting with generalists, the majority of key informants were 50 or more years old. Most key informants $(62 \%)$ were either illiterate or only studied up through elementary school, while only $14.8 \%$ of the generalists settled into this category.

Representing their connection to the land, Visconde de Mauá was the birthplace of most key informants (85\%), while only $22 \%$ of generalists were native (local) to the area. However, most generalists reported living in Visconde de Mauá for at least 10 years and considered themselves familiar with the local landscape dynamics and resources. Southeastern Brazil was the native homeland of $70 \%$ of all respondents, characterizing the regional-centric source of information among interviewees.

Most individuals in both the generalists and key informants groups were engaged in more than one activity as a source of income. Activities related to tourism, such as craftwork commerce and lodging rental, were reported as at least one occupation in $80 \%$ of the respondents.

For most of the key informants, oral transmissions and auditory learning were essential for knowledge acquisition on the use of plants. A great part of such knowledge was acquired by more than one source. The primary information sharers were parents or other relatives $(70 \%)$. However, other local specialists (40\%); books, television and other media (40\%); and spiritual sources (20\%) were also cited.

\section{The use of plant resources}

Two hundred forty plant ethnospecies were identified as cultivated or collected, representing 198 botanical species (Table 2). From the $30^{\text {th }}$ up to the $40^{\text {th }}$ respondent, no further species were added (Figure 2). The species are dis-

Table 2. Collected species $(n=198)$ in different categories of use cited by the community of Visconde de Mauá; Ref = Collection number $(\mathrm{MQ}=$ Mariana Quinteiro); Nat = Native species; Exo = Exotic species; $\mathrm{C}=$ Cultivated species; $\mathrm{E}$ = Extracted species; $\mathrm{N}=$ undetermined. Within Category of Use: Med = Medicinal; Al = Alimentation; Crf = Craftwork; Sym = Symbolic; Cos = Cosmetic; Con = Construction; Fw = Fuel wood; Or = Ornamental.

\begin{tabular}{|c|c|c|c|c|c|}
\hline Species & Local name & Ref. (MQ) & Origin & $\mathrm{C} / \mathrm{E}$ & $\begin{array}{l}\text { Category } \\
\text { of use }\end{array}$ \\
\hline \multicolumn{6}{|l|}{ Acanthaceae } \\
\hline Justicia sp. & Camarão-amarelo & 137 & - & C & Med \\
\hline \multicolumn{6}{|l|}{ Alismataceae } \\
\hline $\begin{array}{l}\text { Echinodorus grandiflorus (Cham. \& Schltdl.) } \\
\text { Micheli }\end{array}$ & Chapéu-de-couro & $96 ; 124$ & Nat & $E$ & Med \\
\hline \multicolumn{6}{|l|}{ Amaranthaceae } \\
\hline $\begin{array}{l}\text { Alternanthera dentata (Moench) Stuchlík } \\
\text { ex R.E.Fr. }\end{array}$ & $\begin{array}{l}\text { Amoxicilina } \\
\text { terramicina trimicina }\end{array}$ & $\begin{array}{l}128 ; 168 \\
209 ; 343\end{array}$ & Nat & C & Med \\
\hline Amaranthus blitum L. & Cariru, caruru & $9 ; 170$ & Exo & $E$ & Med; Al \\
\hline
\end{tabular}


Conservation in Visconde de Mauá, Serra da Mantiqueira, Brazil

\begin{tabular}{|c|c|c|c|c|c|}
\hline Species & Local name & Ref. (MQ) & Origin & $\mathrm{C} / \mathrm{E}$ & $\begin{array}{l}\text { Category } \\
\text { of use }\end{array}$ \\
\hline $\begin{array}{l}\text { Dysphania ambrosioides (L.) Mosyakin \& } \\
\text { Clemants }\end{array}$ & Erva-de-Santa-Maria & $\begin{array}{l}16 ; 73 ; 144 ; \\
221 ; 282\end{array}$ & Nat & $\mathrm{C} / \mathrm{E}$ & Med \\
\hline \multicolumn{6}{|l|}{ Anacardiaceae } \\
\hline Schinus terebinthifolius Raddi & Aroeira & 259 & Nat & $E$ & Med \\
\hline \multicolumn{6}{|l|}{ Apiaceae } \\
\hline Apium sp. & Macelinha & 278 & - & C & Med \\
\hline Centella asiatica (L.) Urb. & Centelha-asiática & 375 & Exo & $\mathrm{E}$ & Med \\
\hline Foeniculum vulgare Mill. & Erva-doce, Funcho & $\begin{array}{l}\text { 56; 116; } \\
\text { 200; 335; } \\
338\end{array}$ & Exo & C & Med \\
\hline Apiaceae sp. 1 & $\begin{array}{l}\text { Agrião-do-seco } \\
\text { Carovinha }\end{array}$ & $94 ; 136$ & - & $\mathrm{C} / \mathrm{E}$ & Med \\
\hline Apiaceae sp. 2 & Erva terrestre & $130 ; 216$ & - & C & Med \\
\hline \multicolumn{6}{|l|}{ Apocynaceae } \\
\hline Asclepias curassavica L. & Erva-braba & 38 & Nat & $E$ & Med \\
\hline \multicolumn{6}{|l|}{ Araceae } \\
\hline Xanthosoma sagittifolium (L.) Schott & Taioba & 250 & Exo & $E$ & Al; Sym \\
\hline \multicolumn{6}{|l|}{ Araucariaceae } \\
\hline Araucaria angustifolia (Bertol.) Kuntze & Pinheiro & 373 & Nat & $E$ & $\begin{array}{l}\text { Con; } \\
\text { Fw; Crf; } \\
\text { Med; Al }\end{array}$ \\
\hline \multicolumn{6}{|l|}{ Aristolochiaceae } \\
\hline Aristolochia sp. & Buta-preta & 184 & - & C & Med \\
\hline \multicolumn{6}{|l|}{ Asteraceae } \\
\hline Achillea millefolium $\mathrm{L}$. & $\begin{array}{l}\text { Artemísia camomila, } \\
\text { mil-rama, novalgina, } \\
\text { macela-canforada, } \\
\text { macelinha, mil- } \\
\text { folhas, ponta-livre, } \\
\text { pronto-alívio }\end{array}$ & $\begin{array}{l}\text { 64; 66; } \\
82 ; 135 ; \\
167 ; 196 ; \\
219 ; 239 ; \\
340\end{array}$ & Exo & C & Med \\
\hline Achyrocline satureioides (Lam.) DC. & Macela, Macelinha & $\begin{array}{l}28 ; 300 ; \\
304 ; 378\end{array}$ & Nat & $E$ & Med; Crf \\
\hline Acmella uliginosa (Sw.) Cass. & Jambu & 165 & Nat & C & $\mathrm{Al}$ \\
\hline Ageratum conyzoides (L.) L. & Erva-de-São João & 255 & Nat & $\mathrm{E}$ & Med \\
\hline Artemisia absinthium L. & Losna & 120 & Exo & $\mathrm{C}$ & Med \\
\hline Artemisia alba Turra & Macela-canforada & 211 & Exo & C & Med \\
\hline Artemisia sp. 1 & Losna-doce & 233 & - & $E$ & Med \\
\hline Artemisia sp. 2 & Losma & 58 & - & C & Med \\
\hline Baccharis pseudomyriocephala Malag. & Carqueja & 202 & Nat & $\mathrm{C}$ & Med \\
\hline Baccharis sp. 1 & Carqueja-amargosa & 92 & - & $E$ & Med \\
\hline Baccharis sp. 2 & \begin{tabular}{|l|} 
Erva-de-Santo \\
Antônio \\
\end{tabular} & 265 & - & $E$ & Med \\
\hline Bidens pilosa L. & Picão, picão-de-praia & $\begin{array}{l}8 ; 25 ; \\
132 ; 267\end{array}$ & Exo & $\mathrm{C} / \mathrm{E}$ & Med \\
\hline
\end{tabular}




\begin{tabular}{|c|c|c|c|c|c|}
\hline Species & Local name & Ref. (MQ) & Origin & C/E & $\begin{array}{l}\text { Category } \\
\text { of use }\end{array}$ \\
\hline Blainvillea sp. & Beldroega & 164 & - & $\mathrm{C}$ & $\mathrm{Al}$ \\
\hline Centratherum punctatum Cass. & Perpétua & 358 & Nat & $E$ & Med \\
\hline Elephantopus mollis Kunth & Fumo-bravo & $27 ; 348$ & Nat & $\mathrm{C} / \mathrm{E}$ & Med \\
\hline $\begin{array}{l}\text { Gymnanthemum amygdalinum (Delile) Sch.Bip. } \\
\text { ex Walp. }\end{array}$ & $\begin{array}{l}\text { Boldo-do-chile, } \\
\text { boldo-de-árvore, } \\
\text { menta-vick, } \\
\text { pariparoba }\end{array}$ & $\begin{array}{l}162 ; 238 ; \\
317\end{array}$ & Exo & $\mathrm{C} / \mathrm{E}$ & Med \\
\hline Matricaria chamomilla L. & Camomila & $\begin{array}{l}67 ; 279 ; \\
316\end{array}$ & Exo & $\mathrm{C}$ & Med \\
\hline Mikania glomerata Spreng. & Guaco & $\begin{array}{l}69 ; 123 ; \\
224 ; 310\end{array}$ & Nat & $\mathrm{C}$ & Med \\
\hline Mikania sp. 1 & $\begin{array}{l}\text { Cipó-cabeludo, } \\
\text { erva-de-São João }\end{array}$ & 314 & - & C & Med \\
\hline Mikania sp. 2 & Macelinha-do-campo & $2 ; 355$ & - & $E$ & Med \\
\hline Mikania sp. 3 & Cipó-cabeludo & 15 & - & $E$ & Med \\
\hline Solidago chilensis Meyen & $\begin{array}{l}\text { Arnica, arnica- } \\
\text { caseira, arnica-de- } \\
\text { horta, arnica-do- } \\
\text { mato, arniquinha }\end{array}$ & $\begin{array}{l}100 ; 206 ; \\
225 ; 298 ; \\
345 ; 351\end{array}$ & Nat & $\mathrm{C} / \mathrm{E}$ & Med \\
\hline Sonchus oleraceus (L.) L. & Serralha & $03 ; 312$ & Nat & $\mathrm{C} / \mathrm{E}$ & Med; Al \\
\hline Taraxacum campylodes G.E.Haglund & Dente-de-leão & $125 ; 237$ & Exo & C/E & Med; Al \\
\hline Vernonanthura phosphorica (Vell.) H.Rob. & Assa-peixe & $\begin{array}{l}34 ; 139 ; \\
268\end{array}$ & Nat & $\mathrm{C} / \mathrm{E}$ & Med \\
\hline Vernonia sp. 1 & Cambará & 235 & - & $E$ & Med \\
\hline Vernonia sp. 2 & Candeia & 179 & - & C & $\begin{array}{l}\text { Con; } \\
\text { Com }\end{array}$ \\
\hline Asteraceae sp. 1 & Arnica-branca & 41 & - & $E$ & Med \\
\hline Asteraceae sp. 2 & $\begin{array}{l}\text { Erva-de-Santo } \\
\text { Antônio }\end{array}$ & 36 & - & $E$ & Med \\
\hline Asteraceae sp. 3 & Batata-yacon & $115 ; 306$ & - & $\mathrm{C}$ & Med; Al \\
\hline Asteraceae sp. 4 & $\begin{array}{l}\text { Boldo-de-árvore, } \\
\text { cambará }\end{array}$ & $103 ; 342$ & - & $\mathrm{C} / \mathrm{E}$ & Med; Con \\
\hline Asteraceae sp. 5 & $\begin{array}{l}\text { Arnica-do-campo, } \\
\text { mata-pasto }\end{array}$ & 370 & - & $E$ & Med \\
\hline Asteraceae sp. 6 & Losma & 46 & - & $\mathrm{C}$ & Med \\
\hline Asteraceae sp. 7 & Artemísia & 70 & - & C & Med \\
\hline \multicolumn{6}{|l|}{ Balsaminaceae } \\
\hline Impatiens walleriana Hook.f. & Beijo-branco & 276 & Exo & $\mathrm{C} / \mathrm{E}$ & Med; Or \\
\hline \multicolumn{6}{|l|}{ Bignoniaceae } \\
\hline Jacaranda mimosifolia D.Don & Jacarandá-mimoso & 377 & Exo & $E$ & Crf \\
\hline Sparattosperma leucanthum (Vell.) K.Schum. & Azeitona-preta & 363 & Nat & $E$ & Med \\
\hline Bignoniaceae sp. 1 & $\begin{array}{l}\text { Cinco-folhas- } \\
\text { do-pequeno, } \\
\text { cinco-folhas }\end{array}$ & 39 & - & $E$ & Med \\
\hline
\end{tabular}


Conservation in Visconde de Mauá, Serra da Mantiqueira, Brazil

\begin{tabular}{|c|c|c|c|c|c|}
\hline Species & Local name & Ref. (MQ) & Origin & C/E & $\begin{array}{l}\text { Category } \\
\text { of use }\end{array}$ \\
\hline \multicolumn{6}{|l|}{ Boraginaceae } \\
\hline Borago officinalis L. & Borragem & 181 & Exo & $\mathrm{C}$ & $\mathrm{Al}$ \\
\hline Symphytum officinale L. & Confrei & $\begin{array}{l}55 ; 166 ; \\
230\end{array}$ & Exo & C & Med; Al \\
\hline \multicolumn{6}{|l|}{ Brassicaceae } \\
\hline Brassica oleracea L. & Couve & 223 & Exo & $\mathrm{C}$ & $\mathrm{Al}$ \\
\hline Nasturtium officinale R.Br, & Agrião & 11 & Exo & $\mathrm{C} / \mathrm{E}$ & Med; Al \\
\hline \multicolumn{6}{|l|}{ Commelinaceae } \\
\hline Commelina sp. & Capueraba & 270 & - & $E$ & Med \\
\hline \multicolumn{6}{|l|}{ Convolvulaceae } \\
\hline Cuscuta sp. & Cipó-seda & 95 & - & $E$ & Med \\
\hline \multicolumn{6}{|l|}{ Costaceae } \\
\hline Costus sp. & $\begin{array}{l}\text { Cana-do-brejo, } \\
\text { caninha-de-macaco, } \\
\text { caninha-do-brejo }\end{array}$ & $\begin{array}{l}72 ; 129 \\
174\end{array}$ & - & C & Med \\
\hline \multicolumn{6}{|l|}{ Crassulaceae } \\
\hline Bryophyllum pinnatum (Lam.) Oken & $\begin{array}{l}\text { Fortuna, saião, } \\
\text { saia-de-remédio }\end{array}$ & $104 ; 108$ & Exo & $E$ & Med \\
\hline Kalanchoe sp. & Saião, saião-do-muro & $\begin{array}{l}81 ; 205 \\
212 ; 253\end{array}$ & - & $\mathrm{C} / \mathrm{E}$ & Med \\
\hline Sedum sp. & Baço-gordo, bálsamo & $\begin{array}{l}111 ; 178 \\
326\end{array}$ & - & C & Med; Al \\
\hline \multicolumn{6}{|l|}{ Cyperaceae } \\
\hline Eleocharis sp. & Cavalinha-do-campo & 5 & - & $E$ & Med \\
\hline \multicolumn{6}{|l|}{ Cucurbitaceae } \\
\hline Sechium edule (Jacq.) Sw. & Chuchu & 281 & Exo & C & Med; Al \\
\hline \multicolumn{6}{|l|}{ Cupressaceae } \\
\hline Cupressus sp. & Cedrinho & 75 & - & C & Med; Con \\
\hline \multicolumn{6}{|l|}{\begin{tabular}{|l|} 
Dennstaedtiaceae \\
\end{tabular}} \\
\hline Pteridium arachnoideum (Kaulf.) Maxon & Samambaia & 23 & Nat & $E$ & Med \\
\hline \multicolumn{6}{|l|}{ Equisetaceae } \\
\hline Equisetum hyemale L. & $\begin{array}{l}\text { Cavalinha, } \\
\text { cavalinha-de-horta }\end{array}$ & $\begin{array}{l}62 ; 105 ; \\
172\end{array}$ & Exo & C & Med \\
\hline \multicolumn{6}{|l|}{ Euphorbiaceae } \\
\hline Croton floribundus Spreng. & Capixinguinha & 294 & Nat & $E$ & Sym \\
\hline Croton lundianus (Didr.) Müll.Arg. & Vassoura-de-cabrito & $29 ; 275$ & Nat & $\mathrm{E}$ & Med \\
\hline Ricinus communis $\mathrm{L}$. & Mamona & $37 ; 254$ & Exo & $E$ & $\begin{array}{l}\text { Med; } \\
\text { Sym }\end{array}$ \\
\hline \multicolumn{6}{|l|}{ Fabaceae } \\
\hline Calliandra sp. & Esponjinha & 51 & - & $\mathrm{C}$ & Con \\
\hline Clitoria sp. & Favinha & 90 & - & $E$ & Med \\
\hline Desmodium adscendens (Sw.) DC. & Favinha, pastelzinho & 357 & Exo & $\mathrm{E}$ & Med \\
\hline Erythrina sp. & Muxoco, suinã & 293 & - & $E$ & Med \\
\hline
\end{tabular}




\begin{tabular}{|c|c|c|c|c|c|}
\hline Species & Local name & Ref. (MQ) & Origin & $\mathrm{C} / \mathrm{E}$ & $\begin{array}{l}\text { Category } \\
\text { of use }\end{array}$ \\
\hline Ormosia arborea (Vell.) Harms & Olho-de-cabra & 380 & Nat & $E$ & Crf \\
\hline Ormosia altimontana J.E.Meireles \& H.C.Lima & Angelim & 374 & Nat & $E$ & Crf; Sym \\
\hline Indigofera suffruticosa Mill. & Anil & 297 & Nat & $\mathrm{E}$ & Med \\
\hline Senna sp. & Pedregoso & 89 & - & $\mathrm{E}$ & Med \\
\hline \multicolumn{6}{|l|}{ Geraniaceae } \\
\hline Pelargonium sp. & Malva & 199 & - & $\mathrm{C}$ & $\begin{array}{l}\text { Con; } \\
\text { Sym; Cos }\end{array}$ \\
\hline \multicolumn{6}{|l|}{ Ginkgoaceae } \\
\hline Ginkgo biloba L. & Gingko biloba & 185 & Exo & C & Med \\
\hline \multicolumn{6}{|l|}{ Lamiaceae } \\
\hline Hyptis radicans (Pohl) Harley \& J.F.B.Pastore & Hortelã-bravo & $\begin{array}{l}1 ; 247 ; \\
319 ; 364\end{array}$ & Nat & $E$ & Med \\
\hline Hyptis sp. & Hortelã-do-mato & 296 & - & $E$ & Med \\
\hline Lavandula angustifolia Mill. & Alfazema & 86 & Exo & C & Med \\
\hline Leonurus sibiricus L. & $\begin{array}{l}\text { Isope macaé, rama- } \\
\text { de-mamangava, sôpe }\end{array}$ & $\begin{array}{l}107 ; 188 \\
256 ; 3 ; 44\end{array}$ & Exo & $\mathrm{C} / \mathrm{E}$ & $\begin{array}{l}\text { Med; } \\
\text { Sym }\end{array}$ \\
\hline Melissa officinalis L. & $\begin{array}{l}\text { Erva-jurema, } \\
\text { grimonha, } \\
\text { jurema, picão }\end{array}$ & $\begin{array}{l}97 ; 134 \\
329\end{array}$ & Exo & C & $\begin{array}{l}\text { Med; } \\
\text { Sym }\end{array}$ \\
\hline Mentha pulegium $\mathrm{L}$ & $\begin{array}{l}\text { Alecrim, poejo, } \\
\text { poejo-caseiro, } \\
\text { poejo-de-horta, } \\
\text { poejo-menta }\end{array}$ & $\begin{array}{l}63 ; 141 ; \\
215 ; 323 ; \\
331 ; 337\end{array}$ & Exo & C & Med \\
\hline Mentha $\times$ piperita $\mathrm{L}$. & $\begin{array}{l}\text { Alevante, boldo- } \\
\text { do-Chile, menta- } \\
\text { do-mato, vick }\end{array}$ & $\begin{array}{l}161 ; 191 \\
252 ; 313 \\
341\end{array}$ & Exo & $\mathrm{C} / \mathrm{E}$ & $\begin{array}{l}\text { Med; } \\
\text { Sym }\end{array}$ \\
\hline Mentha sp. 1 & Hortelã-bravo & 112 & Exo & $E$ & Med \\
\hline Mentha sp. 2 & Hortelã-diferente & 246 & Exo & $E$ & Med \\
\hline Ocimum basilicum L. & Manjericão & 127 & Exo & C & Med; Al \\
\hline Ocimum gratissimum $\mathrm{L}$. & $\begin{array}{l}\text { Alfavaca, alfavaca- } \\
\text { de-árvore }\end{array}$ & $\begin{array}{l}84 ; 226 \\
308\end{array}$ & Exo & C & Med \\
\hline Ocimum sp. 1 & $\begin{array}{l}\text { Alfavaca, aniz, } \\
\text { atroveran, vick }\end{array}$ & $\begin{array}{l}60 ; 117 ; \\
159 ; 208 ; \\
251 ; 283 ; \\
303 ; 315 ; \\
356\end{array}$ & Exo & $\mathrm{C} / \mathrm{E}$ & Med \\
\hline Ocimum sp. 2 & Manjericão & 220 & Exo & $\mathrm{C}$ & Med; Al \\
\hline Origanum vulgare L. & Manjerona & $\begin{array}{l}54 ; 198 \\
218\end{array}$ & Exo & C & $\mathrm{Al}$ \\
\hline Plectranthus neochilus Schltr. & $\begin{array}{l}\text { Boldinho-do-Chile, } \\
\text { Boldo-do-Chile }\end{array}$ & $\begin{array}{l}83 ; 203 ; \\
210 ; 305\end{array}$ & Exo & C & Med \\
\hline Rosmarinus officinalis L. & Alecrim & $77 ; 113$ & Exo & C & Med; Al \\
\hline Salvia officinalis L. & Sálvia-miúda & 78 & Exo & $\mathrm{C}$ & Med \\
\hline Stachys byzantina K.Koch & Sálvia-peluda & $\begin{array}{l}76 ; 109 ; \\
332\end{array}$ & Exo & C & Med \\
\hline
\end{tabular}


Conservation in Visconde de Mauá, Serra da Mantiqueira, Brazil

\begin{tabular}{|c|c|c|c|c|c|}
\hline Species & Local name & Ref. (MQ) & Origin & C/E & $\begin{array}{l}\text { Category } \\
\text { of use }\end{array}$ \\
\hline Tetradenia riparia (Hochst.) Codd & Mirra & 193 & Exo & C & Sym \\
\hline Vitex sp. & $\begin{array}{l}\text { Azeitona-do-mato, } \\
\text { cinco-folhas, tarumã }\end{array}$ & 352 & - & $E$ & Med \\
\hline Lamiaceae sp. 1 & Alevante & $74 ; 228$ & - & $\mathrm{C}$ & Med \\
\hline Lamiaceae sp. 2 & $\begin{array}{l}\text { Cidreira, erva- } \\
\text { cidreira, erva- } \\
\text { cidreira-limão }\end{array}$ & $\begin{array}{l}57 ; 190 \\
318\end{array}$ & - & C & Med \\
\hline Lamiaceae sp. 3 & $\begin{array}{l}\text { Alevante, hortelã, } \\
\text { hortelã-do-escuro, } \\
\text { hortelã-pretinho }\end{array}$ & $\begin{array}{l}68 ; 143 \\
322\end{array}$ & - & C & Med \\
\hline Lamiaceae sp. 4 & Hortelã & 249 & - & $E$ & Med \\
\hline Lamiaceae sp. 5 & Lavanda & 194 & - & C & Sym \\
\hline Lamiaceae sp. 6 & Menta & 85 & - & C & Med \\
\hline Lamiaceae sp. 7 & Menta & 189 & - & C & Med; Al \\
\hline \multicolumn{6}{|l|}{ Lauraceae } \\
\hline Laurus nobilis L. & Louro & 207 & Exo & C & $\mathrm{Al}$ \\
\hline Persea americana Mill. & Abacate & $217 ; 277$ & Exo & C & $\begin{array}{l}\text { Med; Al; } \\
\text { Cos }\end{array}$ \\
\hline Lauraceae sp. & Canela & 176 & - & C & Med \\
\hline \multicolumn{6}{|l|}{ Loranthaceae } \\
\hline Struthanthus sp. & Erva-de-passarinho & $33 ; 231$ & Nat & $E$ & Med \\
\hline Loranthaceae sp. & Erva-de-passarinho & 327 & - & $E$ & Med \\
\hline \multicolumn{6}{|l|}{ Lythraceae } \\
\hline Cuphea sp. 1 & $\begin{array}{l}\text { Pé-de-pombo, } \\
\text { sete-sangrias }\end{array}$ & $\begin{array}{l}101 ; 118 \\
213 ; 361\end{array}$ & Exo & $\mathrm{C} / \mathrm{E}$ & Med \\
\hline \multicolumn{6}{|l|}{ Malvaceae } \\
\hline Gossypium hirsutum L. & Algodão & $\begin{array}{l}169 ; 244 ; \\
284\end{array}$ & Exo & $E$ & Med \\
\hline Malvaviscus arboreus Cav. & Hibisco & $\begin{array}{l}47 ; 195 \\
372\end{array}$ & Exo & C & $\begin{array}{l}\text { Med; Or; } \\
\text { Con; Sym }\end{array}$ \\
\hline \multicolumn{6}{|l|}{ Melastomataceae } \\
\hline Melastomataceae sp. 1 & Mexerica, Mexerico & \begin{tabular}{|l|}
$274 ; 347 ;$ \\
371 \\
\end{tabular} & - & $E$ & Med; Al \\
\hline Melastomataceae sp. 2 & Mexerico & 13 & - & $E$ & Med \\
\hline \multicolumn{6}{|l|}{ Moraceae } \\
\hline Ficus carica L. & Figo & $126 ; 229$ & Exo & C & $\begin{array}{l}\text { Al; Med; } \\
\text { Sym }\end{array}$ \\
\hline $\begin{array}{l}\text { Sorocea bonplandii (Baill.) W.C.Burger, Lanj. \& } \\
\text { Wess.Boer }\end{array}$ & Espinheira-santa & $\begin{array}{l}22 ; 177 ; \\
242 ; 360\end{array}$ & Nat & $E$ & Med \\
\hline \multicolumn{6}{|l|}{ Myrtaceae } \\
\hline Eucalyptus sp. & Eucalipto & 380 & Exo & $E$ & $\begin{array}{l}\text { Med; } \\
\text { Con; Fw }\end{array}$ \\
\hline Eugenia uniflora L. & Pitanga & $119 ; 307$ & Nat & C & Al; Med \\
\hline Plinia cauliflora (Mart.) Kausel & Jaboticaba & 52 & Nat & C & $\mathrm{Al} ; \mathrm{Crf}$ \\
\hline
\end{tabular}




\begin{tabular}{|c|c|c|c|c|c|}
\hline Species & Local name & Ref. (MQ) & Origin & C/E & $\begin{array}{l}\text { Category } \\
\text { of use }\end{array}$ \\
\hline Psidium guajava L. & Goiaba & 232 & Exo & $\mathrm{C}$ & Al; Med \\
\hline Psidium sp. & Araçá-do-campo & 302 & - & $\mathrm{C}$ & Med \\
\hline \multicolumn{6}{|l|}{ Nyctaginaceae } \\
\hline Mirabilis jalapa L. & Maravilha & 173 & Exo & $\mathrm{C}$ & Cos \\
\hline \multicolumn{6}{|l|}{ Oleaceae } \\
\hline Jasminum polyanthum Franch. & Jasmim & 336 & Exo & C & $\begin{array}{l}\text { Med; } \\
\text { Sym; } \\
\text { Con; Or }\end{array}$ \\
\hline \multicolumn{6}{|l|}{ Passifloraceae } \\
\hline Passiflora edulis Sims & Maracujazinho & 24 & Nat & $E$ & $\begin{array}{l}\text { Al; Med; } \\
\text { Crf }\end{array}$ \\
\hline \multicolumn{6}{|l|}{ Phytolaccaceae } \\
\hline Petiveria alliacea $\mathrm{L}$. & Guiné & $234 ; 261$ & Nat & $E$ & $\begin{array}{l}\text { Med; } \\
\text { Sym }\end{array}$ \\
\hline \multicolumn{6}{|l|}{ Phyllanthaceae } \\
\hline Phyllanthus tenellus Roxb. & $\begin{array}{l}\text { Erva-pombinho, } \\
\text { quebra-pedra }\end{array}$ & $\begin{array}{l}50 ; 114 ; \\
187 ; 241\end{array}$ & Nat & $\mathrm{C} / \mathrm{E}$ & Med \\
\hline \multicolumn{6}{|l|}{ Piperaceae } \\
\hline Piper umbellatum L. & Capeba, pau-peroba & $122 ; 309$ & Nat & C & Med \\
\hline Piper sp. 1 & Jaborandi & 269 & - & $\mathrm{E}$ & Cos \\
\hline Piper sp. 2 & Jaborandi & 350 & - & $E$ & Med \\
\hline Piper sp. 3 & Jaborandi & 14 & - & $E$ & Cos; Med \\
\hline \multicolumn{6}{|l|}{ Plantaginaceae } \\
\hline Digitalis purpurea L. & Digitalis & 183 & Exo & C & Med \\
\hline Plantago sp. & Trançagem & $20 ; 240$ & - & $E$ & Med \\
\hline Scoparia dulcis L. & $\begin{array}{l}\text { Vassoura-branca, } \\
\text { vassoura-de-São } \\
\text { Pedro, vassourinha- } \\
\text { do- campo }\end{array}$ & $\begin{array}{l}19 ; 262 ; \\
365\end{array}$ & Nat & $E$ & $\begin{array}{l}\text { Med; } \\
\text { Sym }\end{array}$ \\
\hline \multicolumn{6}{|l|}{ Platanaceae } \\
\hline Platanus acerifolia (Aiton) Willd. & Maple, plátano & 182 & Exo & C & Med \\
\hline \multicolumn{6}{|l|}{ Poaceae } \\
\hline Coix lacryma-jobi L. & $\begin{array}{l}\text { Lágrima-de- } \\
\text { nossa senhora }\end{array}$ & 131 & Exo & C & Med \\
\hline Cymbopogon citratus (DC.) Stapf & Capim-limão & $61 ; 321$ & Exo & C & Med; Crf \\
\hline $\begin{array}{l}\text { Cymbopogon flexuosus (Nees ex Steud.) } \\
\text { W.Watson }\end{array}$ & Citronela & $87 ; 204$ & Exo & C & Med \\
\hline Melinis minutiflora P.Beauv. & Capim-gordura & 6 & Exo & $E$ & Med \\
\hline Poaceae sp. & Bambu & 32 & - & $E$ & $\begin{array}{l}\text { Crf; Med; } \\
\text { Sym; } \\
\text { Cos; Al }\end{array}$ \\
\hline \multicolumn{6}{|l|}{ Polygalaceae } \\
\hline Polygala paniculata L. & $\begin{array}{l}\text { Aguiné, Guiné, } \\
\text { Guinezinho }\end{array}$ & $17 ; 271$ & Nat & $E$ & $\begin{array}{l}\text { Med; } \\
\text { Sym }\end{array}$ \\
\hline
\end{tabular}


Conservation in Visconde de Mauá, Serra da Mantiqueira, Brazil

\begin{tabular}{|c|c|c|c|c|c|}
\hline Species & Local name & Ref. (MQ) & Origin & C/E & $\begin{array}{l}\text { Category } \\
\text { of use }\end{array}$ \\
\hline \multicolumn{6}{|l|}{ Polygonaceae } \\
\hline Persicaria hydropiperoides (Michx.) Small & $\begin{array}{l}\text { Erva-de- bicho, } \\
\text { ramaim }\end{array}$ & $\begin{array}{l}93 ; 145 ; \\
263 ; 273 ; \\
311\end{array}$ & Nat & $\mathrm{C} / \mathrm{E}$ & Med \\
\hline \multicolumn{6}{|l|}{ Polypodiaceae } \\
\hline Microgramma squamulosa (Kaulf.) de la Sota & Cipó-índio & 43 & Nat & $E$ & Med \\
\hline Serpocaulon fraxinifolium (Jacq.) A.R.Sm. & Samambaia & 243 & Nat & $E$ & Med \\
\hline \multicolumn{6}{|l|}{ Pteridaceae } \\
\hline Adiantum raddianum C.Presl & Avenca & $\begin{array}{l}26 ; 91 ; 146 ; \\
214 ; 330\end{array}$ & Nat & C & Med \\
\hline \multicolumn{6}{|l|}{ Rosaceae } \\
\hline Eriobotrya japonica (Thunb.) Lindl. & Ameixa-amarela & 399 & Exo & $E$ & $\mathrm{Al}$ \\
\hline Prunus persica (L.) Batsch & Pêssego & 48 & Exo & C & $\mathrm{Al} ; \mathrm{Crf}$ \\
\hline Rosa sp. 1 & Rosa-branca & $227 ; 334$ & - & $\mathrm{C}$ & $\begin{array}{l}\text { Med; } \\
\text { Sym; Or }\end{array}$ \\
\hline Rosa sp. 2 & Rosa-branca & 53 & - & $\mathrm{C}$ & Med; Or \\
\hline Rubus brasiliensis Mart. & Amora & 18 & Nat & $E$ & $\begin{array}{l}\text { Al; Med; } \\
\text { Crf }\end{array}$ \\
\hline Rubus rosifolius Sm. & Amora-do-campo & $30 ; 257$ & Nat & $E$ & $\begin{array}{l}\text { Med; } \\
\text { Cos; Crf }\end{array}$ \\
\hline Rubus sellowii Cham. \& Schltdl. & $\begin{array}{l}\text { Amorinha-preta, } \\
\text { framboesa }\end{array}$ & 368 & Nat & $E$ & Med; Crf \\
\hline \multicolumn{6}{|l|}{ Rubiaceae } \\
\hline Coffea arabica L. & Café & 49 & Exo & C & Al; Crf \\
\hline Spermacoce verticillata L. & $\begin{array}{l}\text { Cordão-de-frade, } \\
\text { cordão-de-são } \\
\text { francisco, serralha }\end{array}$ & $3 ; 272 ; 354$ & Nat & $E$ & Al; Med \\
\hline Rubiaceae sp. 1 & Poejo-branco & 4 & - & $E$ & Med \\
\hline Rubiaceae sp. 2 & Boçorão-de-boi & 7 & - & $E$ & Med \\
\hline \multicolumn{6}{|l|}{ Rutaceae } \\
\hline Citrus sp. 1 & Laranja & 333 & - & C & Med \\
\hline Citrus sp. 2 & Limão-cravo & 40 & - & $E$ & Al; Med \\
\hline Ruta graveolens L. & Arruda & $80 ; 197$ & Exo & $\mathrm{C}$ & $\begin{array}{l}\text { Med; } \\
\text { Sym }\end{array}$ \\
\hline Zanthoxylum sp. & $\begin{array}{l}\text { Mamica-de-cadela, } \\
\text { mamica-de-porca }\end{array}$ & 367 & - & $E$ & Med \\
\hline \multicolumn{6}{|l|}{ Sapindaceae } \\
\hline Cupania sp. & Quina-rosa & 353 & - & $E$ & Med \\
\hline \multicolumn{6}{|l|}{ Scrophulariaceae } \\
\hline Buddleja stachyoides Cham. \& Schltdl. & $\begin{array}{l}\text { Babaço, barbacea, } \\
\text { erva-de-Santo } \\
\text { Antônio, verbasco }\end{array}$ & $\begin{array}{l}35 ; 98 ; 266 ; \\
346 ; 379\end{array}$ & Nat & $E$ & Med \\
\hline \multicolumn{6}{|l|}{ Smilacaceae } \\
\hline Smilax sp. 1 & Japecanga & 44 & - & $E$ & Med \\
\hline
\end{tabular}




\begin{tabular}{|c|c|c|c|c|c|}
\hline Species & Local name & Ref. (MQ) & Origin & $\mathrm{C} / \mathrm{E}$ & $\begin{array}{l}\text { Category } \\
\text { of use }\end{array}$ \\
\hline Smilax sp. 2 & Salsaparrilha & 31 & - & $E$ & Med \\
\hline \multicolumn{6}{|l|}{ Solanaceae } \\
\hline $\begin{array}{l}\text { Brugmansia suaveolens (Humb. \& Bonpl. } \\
\text { ex Willd.) Sweet }\end{array}$ & Trombeta & 376 & Exo & $E$ & Med \\
\hline Physalis angulata L. & Joapoga & 366 & Exo & $\mathrm{E}$ & Med \\
\hline Solanum americanum Mill. & \begin{tabular}{|l|} 
Erva-moura, \\
pimenta-de-sapo \\
\end{tabular} & $10 ; 71$ & Nat & $\mathrm{C} / \mathrm{E}$ & Med \\
\hline Solanum sp. 1 & Arrebenta-cavalo & 245 & - & $\mathrm{E}$ & Med \\
\hline Solanum sp. 2 & Jubebinha, jurubeba & $175 ; 280$ & - & C & Med \\
\hline Solanum sp. 3 & Capeba-jurubeba & $21 ; 42 ; 102$ & - & $E$ & Med \\
\hline Vassobia breviflora (Sendtn.) Hunz. & Mamona & 254 & Nat & $E$ & $\begin{array}{l}\text { Med; } \\
\text { Sym }\end{array}$ \\
\hline \multicolumn{6}{|l|}{ Tropaeolaceae } \\
\hline Tropaeolum majus L. & Chagas & 320 & Exo & C & Al; Med \\
\hline \multicolumn{6}{|l|}{\begin{tabular}{|l|} 
Urticaceae \\
\end{tabular}} \\
\hline Cecropia hololeuca Miq. & Imbaúba & 299 & Nat & $E$ & Med \\
\hline Cecropia sp. & Imbaúa & 359 & - & $E$ & Med \\
\hline \multicolumn{6}{|l|}{ Verbenaceae } \\
\hline Lippia alba (Mill.) N.E.Br. ex Britton \& P.Wilson & $\begin{array}{l}\text { Cidreira-de- árvore, } \\
\text { Cidreira-de-nossa } \\
\text { senhora, melissa, } \\
\text { melissa-verdadeira } \\
\end{array}$ & $\begin{array}{l}65 ; 171 \\
201 ; 260\end{array}$ & Nat & $\mathrm{C} / \mathrm{E}$ & Med \\
\hline Stachytarpheta sp. & $\begin{array}{l}\text { Gerbão, gervão, } \\
\text { gervão-roxo, jeribom }\end{array}$ & $\begin{array}{l}59 ; 292 ; \\
236 ; 369 \\
\end{array}$ & - & $\mathrm{C} / \mathrm{E}$ & Med \\
\hline \multicolumn{6}{|l|}{ Violaceae } \\
\hline Anchietea pyrifolia (Mart.) G.Don & Cipó-sumi & 12 & Nat & $E$ & Med \\
\hline Viola odorata L. & Chagas & 339 & Exo & $\mathrm{C}$ & Med \\
\hline \multicolumn{6}{|l|}{ Vitaceae } \\
\hline Cissus verticillata (L.) Nicolson \& C.E.Jarvis & Insulina & 140 & Nat & C & Med \\
\hline Cissus sp. 1 & Uva-do-mato & 349 & - & $E$ & Med \\
\hline Cissus sp. 2 & Fava-de-Santo Inácio & 295 & - & $E$ & Med \\
\hline \multicolumn{6}{|l|}{ Zingiberaceae } \\
\hline Alpinia zerumbet (Pers.) B.L.Burtt \& R.M.Sm. & Alfazema, colônia & 106 & Exo & C & $\begin{array}{l}\text { Med; } \\
\text { Sym }\end{array}$ \\
\hline Curcuma longa L. & Açafrão & 110 & Exo & C & Al; Med \\
\hline Zingiber officinale Roscoe & $\begin{array}{l}\text { Gengibre, raiz- } \\
\text { da-vida }\end{array}$ & 138 & Exo & C & Med \\
\hline Zingiberaceae sp. 1 & Bastão & 362 & - & $E$ & Med \\
\hline \multicolumn{6}{|l|}{ Indeterminate } \\
\hline sp. 1 & Arnica-de-árvore & 142 & - & C & Med \\
\hline sp. 2 & Prímula & 186 & - & $\mathrm{C}$ & Med \\
\hline sp. 3 & Quina-rosa & 301 & - & $E$ & Med \\
\hline sp. 4 & Guatambu & 163 & - & C & Med \\
\hline
\end{tabular}


Quinteiro et al. - Inventory and Implications of Plant Use for Environmental

Conservation in Visconde de Mauá, Serra da Mantiqueira, Brazil

\begin{tabular}{|l|l|l|l|l|l|}
\hline Species & Local name & Ref. (MQ) & Origin & C/E & $\begin{array}{l}\text { Category } \\
\text { of use }\end{array}$ \\
\hline sp. 5 & Rosa-mosqueta & 180 & - & C & Con \\
\hline sp. 6 & Agrimoni & 121 & - & C & $\mathrm{Al}$; Med \\
\hline sp. 7 & Cedro & 99 & - & E & Con; Med \\
\hline sp. 8 & Hortelã-menta, menta & 133 & - & C & Med \\
\hline sp. 9 & Azedinho & 248 & - & E & Al \\
\hline sp. 10 & Patchouli & 192 & - & C & Sym \\
\hline
\end{tabular}

tributed in 61 families, especially Asteraceae, Lamiaceae, and Fabaceae (Figure 3).

Among the plants identified to the species level, there was near equality between native (45\%) and exotic (55\%) species. Furthermore, $44 \%$ of the species could only be found cultivated, and $45 \%$ were discovered via wild collection. The remaining $11 \%$ were found in both cultivated gardens and among wild growth in Visconde de Mauá. Backyard gardens held $55 \%$ of all species and contained at least one species in each verified category of use; most predominantly, medicinal species were found in every back- yard analyzed. Thus, in Visconde de Mauá backyard gardens can be considered as a relevant landscape unit.

\section{Medicinal plants}

The majority of species listed in this study (172) were reportedly used for medicinal purposes. From the species with determined origin, $45 \%$ are native species. The use of medicinal plants does not seem a threat to environmental conservation. The plant parts most used were leaves and aerial stems (87\%), which are regularly available and easily regenerated. Additionally, approximately half of the medicinal species are cultivated.

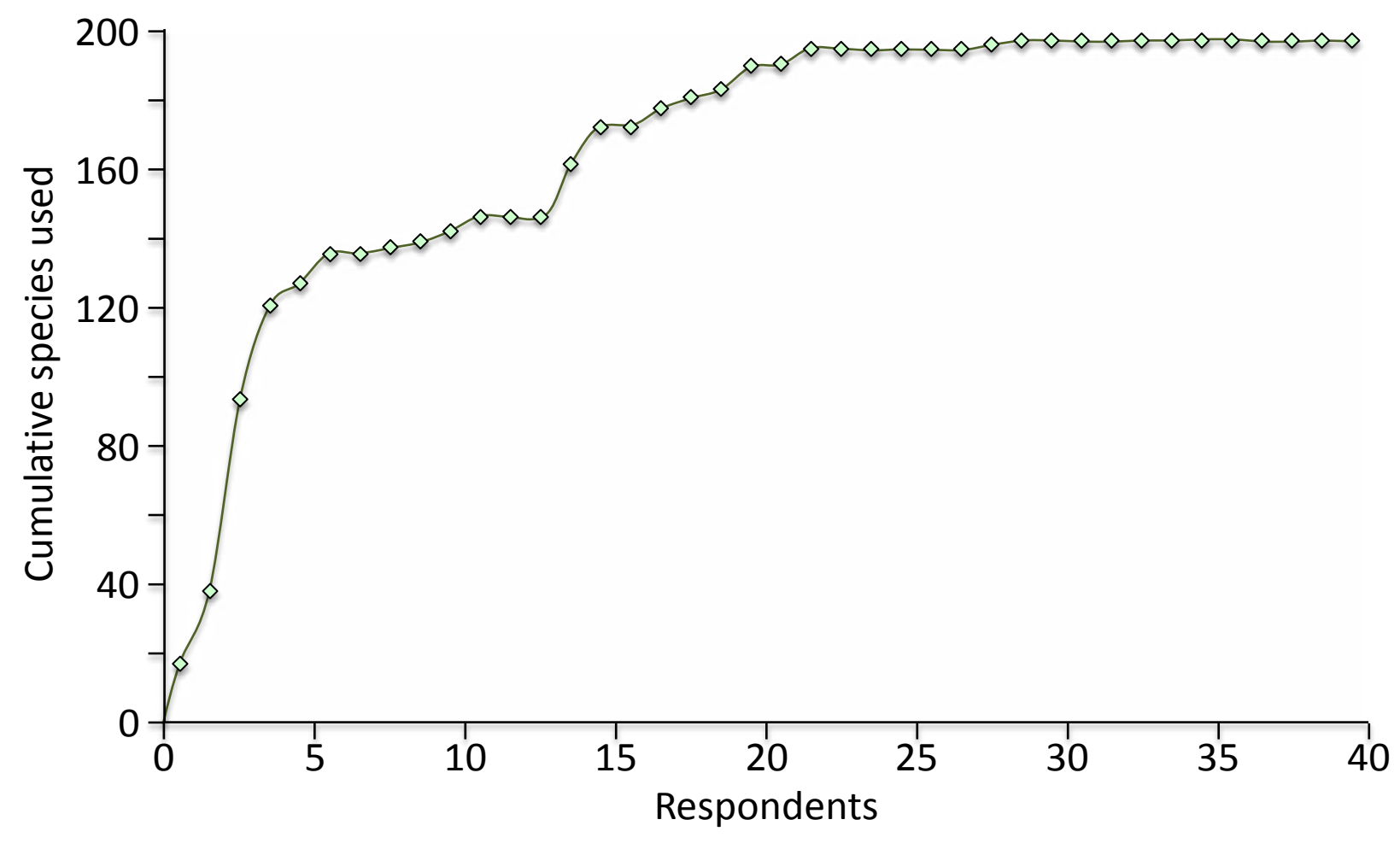

Figure 2. Accumulated frequency of species cited by the respondents from Visconde de Mauá community, Rio de Janeiro, Brazil ( $n=197$ species). 


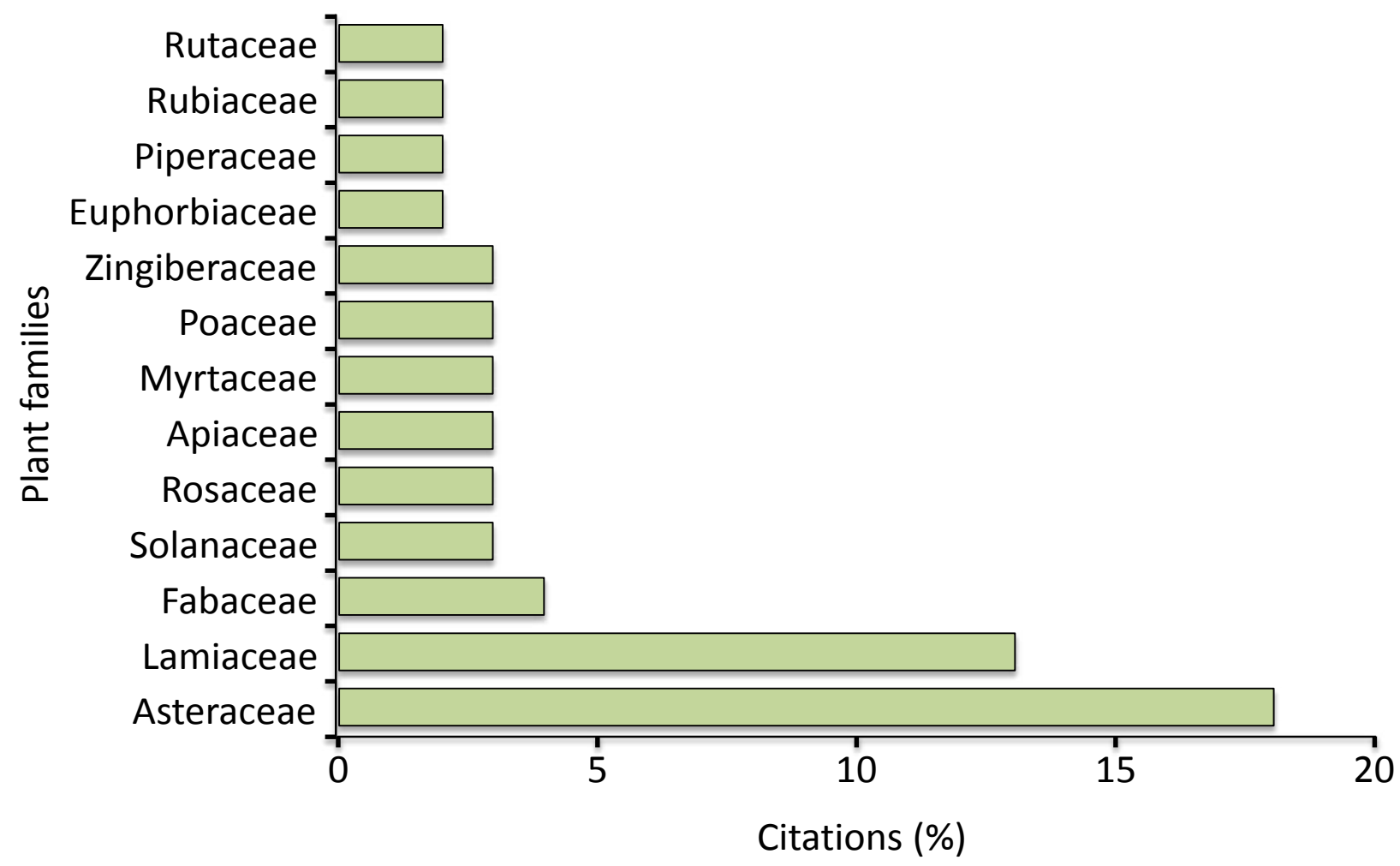

Figure 3. Percentages of plants cited by respondents from Visconde de Mauá, Rio de Janeiro, Brazil, as distributed by plant family.

\section{Plants used for craftwork}

Another prominent category of plant use is craftwork as tourism in Visconde de Mauá pushes trade in local products, especially handmade ones. Half of the respondents make and/or sell craftwork with local plant resources, three of whom were key informants. However, this activity does not seem to be traditional since most artisans we interviewed came from other regions.

The main species used for manufacturing homemade jams, jellies, and brandies were Rubus rosifolius Sm., Rubus brasiliensis Mart., Rubus sellowii Cham. \& Schltdl., Araucaria angustifolia (Bertol.) Kuntze, Plinia cauliflora (Mart.) Kausel, Eriobotrya sp., Passiflora edulis Sims, and Prunus persica (L.) Batsch. Artisanal herbal pillows are made using Achyrocline satureioides (Lam.) DC., Cymbopogon citratus (DC.) Stapf, and Matricaria chamomilla L. For making ornaments and jewelry, the most cited species were Ormosia altimontana J.E.Meireles \& H.C.Lima (85\%), Ormosia arborea (Vell.) Harms (69\%), A. angustifolia (46\%), Poaceae spp. (46\%), P. persica (31\%), Jacaranda mimosifolia D.Don (38\%), and $A$. satureioides $(23 \%)$. The preferential ordering of use of the main artisanal plants were: Ormosia altimontana, O. arborea, $A$. angustifolia, and J. mimosifolia.

\section{Plants used for alimentation}

Though Visconde de Mauá is a rural region, only $35 \%$ of the respondents cultivate food crops. Community members purchase sporadically available products in a few local markets despite their expense. The respondents declared cultivating plants for alimentation is laborious and brings less financial return than tourism-related activities. Additionally, interviewees indicated intent to use fertile spaces capable of plant cultivation to construct new housing structures.

In total, this research categorized 72 species as crops, but only $8 \%$ of these plants were not cultivated, due to being collected directly from the forest and its surroundings. The main extracted species are: Rubus rosifolius, $A$. angustifolia, $P$. edulis, an unidentified bamboo (Poaceae sp.), and Melastomataceae sp. 1 and sp. 2. Some species $(11 \%)$ were obtained by both extraction and cultivation such as Sonchus oleraceus (L.) L., Citrus sp. 2, P. persica, $R$. brasiliensis, $R$. sellowii, Eriobotrya sp., Nasturtium officinale W.T.Aiton, Tropaeolum majus L., and Symphytum officinale L.

Regarding these cultivated vegetables, $63 \%$ were herbaceous, $18 \%$ arboreal, $14 \%$ shrubby, and $5 \%$ climber, showing an overall herbaceous habit predominance. 


\section{Quinteiro et al. - Inventory and Implications of Plant Use for Environmental Conservation in Visconde de Mauá, Serra da Mantiqueira, Brazil}

Interviewees did not mention the use of agrochemicals, pesticides, and industrial fertilizers in crops. As local agriculture is performed in small scale without chemical input, agro-toxins do not threaten the conservation aims of the Environmental Protection Area.

\section{Plants used for construction}

Nine species were reported for construction: Araucaria angustifolia, Jasminum polyanthum Franch., Malvaviscus arboreus Cav., Calliandra sp., Cupressus sp., Eucalyptus sp., Vernonia sp. 2, Asteraceae sp. 4, and one undetermined species (popularly known as cedro). This category includes plants used for construction of housing foundations and anchors (pieces to anchor walls), planks for the floor and ceiling, hedges, cable hoe, and furniture such as tables, sofas, and chairs. Table 2 includes these plants and their functions.

The felling of some of these species is forbidden now by IBAMA, and informants were aware of this restriction. Nevertheless, they reported they still retain the knowledge of how to use these species. Due to its inclusion in the list of Brazilian endangered species, $A$. angustifolia use for construction is prohibited. Correspondingly, civil buildings are predominantly constructed via imported outside wood.

\section{Fuel wood}

Respondents reported using $A$. angustifolia, Eucalyptus sp., and Vernonia sp. 2 for fuel wood in addition to their use in construction. The use of plants as fuel wood requires particular attention, due to the intense local tourism and the climate of the region. Though respondents acknowledged the prohibition on the use of these plants, these species are still commonly found in many hostels and hotels. Representing a conflict between earning income and following the law, selling firewood is a profitable activity in the region as a pack of firewood often found in local markets costs around R $\$ 15.00$ (US\$ 7.50).

\section{Ornamental plants}

This category includes plants used for decorative purposes with the intent to "spruce up" a dwelling, and the majority of plants are species that require little care in backyards or in indoor pots. Ornamental plant use was more pronounced in the inns and hotels as fundamental components in landscaping to attract attention of guests and tourists. The plants most cited for this purpose were Jasminum polyanthum, Impatiens walleriana Hook.f., Adiantum raddianum C.Presl, and two species of Rosaceae (Rosa sp.1 and Rosa sp.2).

\section{Plants of symbolic use}

Plants included in this category are used in superstitions, rituals for healing, and prevention of "cultural illnesses" interpreted as manifestations, and diseases that do not present a reasoned scientific cause. Patients are usually treated via a "blessing of the sick person," prayers performed in the presence of a fresh plant and the Catholic rosary, the use of plants in a sitz bath, and for other treatments.

The baths are taken "neck down," so as not to ward off the person's "guardian angel." In addition, fumigation is carried out with a dried version of the plant, after being placed in containers or directly via burning its branches. These plants can also be planted at the entrance or the back of the house, in pots or gardens, to bring good luck and "not let the evil eye come." Due to the use of plant parts in this category, symbolic plants have cultural importance in Visconde de Mauá.

\section{Discussion}

\section{The respondents and their perceptions on plant resources}

The groupings of informants in this study were similar to those found by Voeks (2007) and Fonseca-Kruel and Peixoto (2004). The relative dominance of women in comparison to men evidences the role of key informant women as guardians of the knowledge on medicinal plants; similar results on women's knowledge about plants were found by Medeiros et al. (2004), Pinto et al. (2006), and Voeks (2007). In general, women had higher enrollment with cultivated plants in backyards and gardens as the practice required less concerted effort. Additionally, a fear of wild animals in the surrounding areas contributed to their preference for plant cultivation. Contrary to domestic cultivation, men focused on collecting medicinal plants in wild fields, guided by popular knowledge. This method has previously been demonstrated to increase the number of native species used for medical purposes by the local rural communities (Pinto et al. 2006).

The advanced age of most informants is consistent with other studies (Fonseca-Kruel \& Peixoto 2004, Pinto et al. 2006, Schardong \& Cervi 2000, Voeks \& Leony 2004). This statistic may be resultant of many young individuals in these communities becoming less interested in understanding local plants and their potential uses. Ethnobotanical knowledge of these informants did not seem to be related to the formal school education, which previous scholarly works indicate sometimes actually hinders it. Di Stasi (1996) and Voeks and Leony (2004) stressed that mandatory education systems seem to be inversely related to empirical knowledge of medicinal plants.

The findings here that a majority of respondents were involved in aspects of the tourism trade are similar to findings of Voeks (2007) when studying the very touristic re- 
gion of Chapada Diamantina in Bahia, Brazil. And the reported predominance of oral transmission among respondents is corroborated by the work of Medeiros et al. (2004) who also emphasized the prevalence of oral information transmission mentioned by their respondents.

Key informants could be characterized as remnants, direct descendants, or followers of local native traditional people (Arruda 1997, Diegues 1996). In Visconde de Mauá, key informants are able to maintain the knowledge from different ethnic groups, especially the local knowledge preserved throughout the generations. They are engaged to preserve traditional cultures and values regarding plants. However, tourism and the accelerated industrialization process may affect the local knowledge and the native flora (Medeiros et al. 2004). The tourism that takes place in Visconde de Mauá may threaten biodiversity in mountain and high mountain rainforest, since in other ecosystems of the Atlantic Forest, farming activities decrease as tourism increases. Correspondingly, cultural and biological diversity are endangered as plant knowledge is already being lost (Hanazaki et al. 2000). The inhabitants' cognitive connection with nature and their oral traditions are perhaps in greater risk of extinction than medicinal plants, which was already stressed by Voeks (2007) for traditional people.

\section{The use of plant resources}

Backyard gardens hold economic, nutritional, and social community characteristics. These systems are germplasm banks that allow cultivation of plants of interest and also the exchange of this material among community members (Albuquerque 1999). The horticultural activities in backyards result in regular production of fruit and medicinal plants, thus, inhabitants reduce their dependence on products acquired externally (Pasa et al. 2005). Despite their importance, backyard gardens have been in decline in Visconde de Mauá due to the conversion of these spaces into hostels, campsites, and small cottages in order to shelter tourists.

\section{Medicinal plants}

The great number of species reported in this category of use reflects the local reality; the population of Visconde de Mauá is far from urban centers, with difficult road access and generally low incomes. The community lacks health services, such as hospitals and ambulances for emergencies, thus the alternative medicinal practices are regular. These practices persist when health services are inaccessible for part of the population (Rezende \& Cocco 2002). Another reason for the preference for treatment based on traditional medicine knowledge is the high cost of allopathic medicines (Medeiros et al. 2004).
The loss of traditional knowledge of medicinal plants is apparent. People ascribe commercial drug names to some of the cultivated medicinal species, as can be seen in amoxilina, terramicina, novalgina, atroveram, and anador. This decline has also happened in other Brazilian regions (Pinto et al. 2006, Voeks 2007).

\section{Plants used for craftwork}

The dissemination of new technologies, increased by globalization and considerable local tourism, seems to seasonally affect the distribution of plants in the categories of use. This is the case of plants used for making homemade jams and jellies. In past times when tourism was not intense in Visconde de Mauá, these plants may have been used exclusively for food. Nowadays, with the tourism-induced seasonal changes in the local economic relations, such products are destined for the local markets in order to create or increase family income. For this reason, these plants were categorized into the commercial craftwork category. Similarly, in traditional communities, plants used for stuffing pillows may be classified within the category "technology" (Fonseca-Kruel \& Peixoto 2004), but in Visconde de Mauá, pillows are produced for sale to tourists and not used in the community. Therefore, these pillows are not considered as a technology employed to their benefit, but as commercial craftwork products that augment the income of the local residents. This therefore represents one alternate categorization of the preferred use of certain plant species to the community throughout its historical and economic process.

The most preferred species in the craftwork category, O. altimontana, is locally known as angelim, caju, cajuzinho, and olho-de-cabra-amarelo. It is an endemic species from Atlantic Forest, and its natural growth is restricted to elevations only above $1000 \mathrm{~m}(3300 \mathrm{ft})$ (Meireles 2014). In Visconde de Mauá, O. altimontana seeds are used to produce ornaments due to its beautiful morphology (Figure 4). Additionally, its endemism in high mountains influences the preference for using this species, which results in unique artisanal products and therefore greater commercial value.

The orange-reddish integument of $O$. altimontana seeds is hard and impermeable, which likely prevents germination. Another species of the genus found in this region, $O$. arborea, has seeds with dormancy imposed by the integument, which is difficult for seedling production (Zamith \& Scarano 2004). The majority of seeds of O. altimontana are collected from the ground, at the time of fruit ripening. Some of the respondents also reported sifting the soil surrounding the trees to collect more seeds from the soil seed bank. Large amounts of seeds are sold at low prices to intermediaries, for resale in large cities. Considering the limited availability, the difficulties in propagation, and also the interference on soil seed bank, the over-exploita- 
Conservation in Visconde de Mauá, Serra da Mantiqueira, Brazil
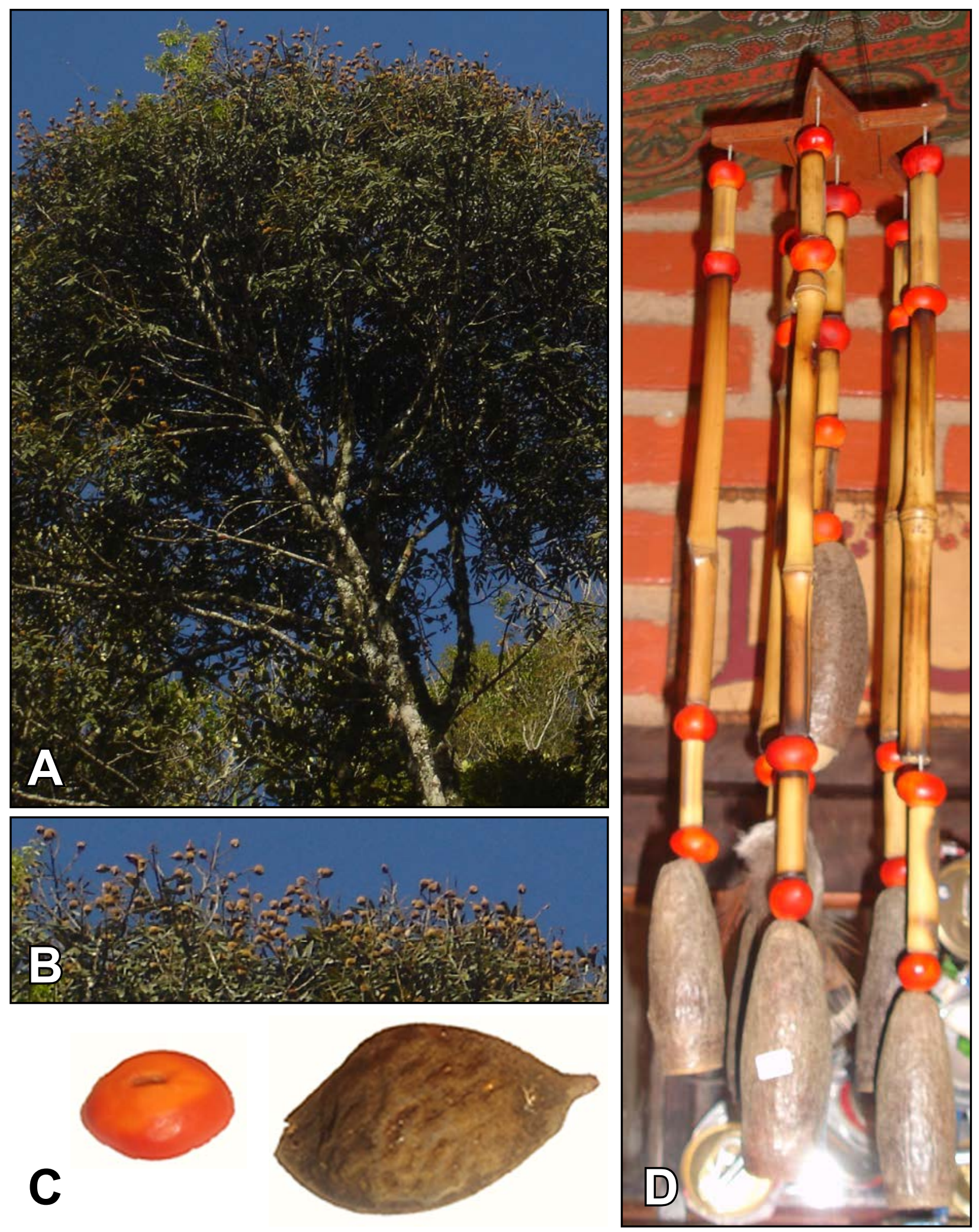

Figure 4. Ormosia altimontana J.E.Meireles \& H.C.Lima (A), tree with fruit (B), fruit and seed (C), and craftwork using O. altimontana seeds (D). 

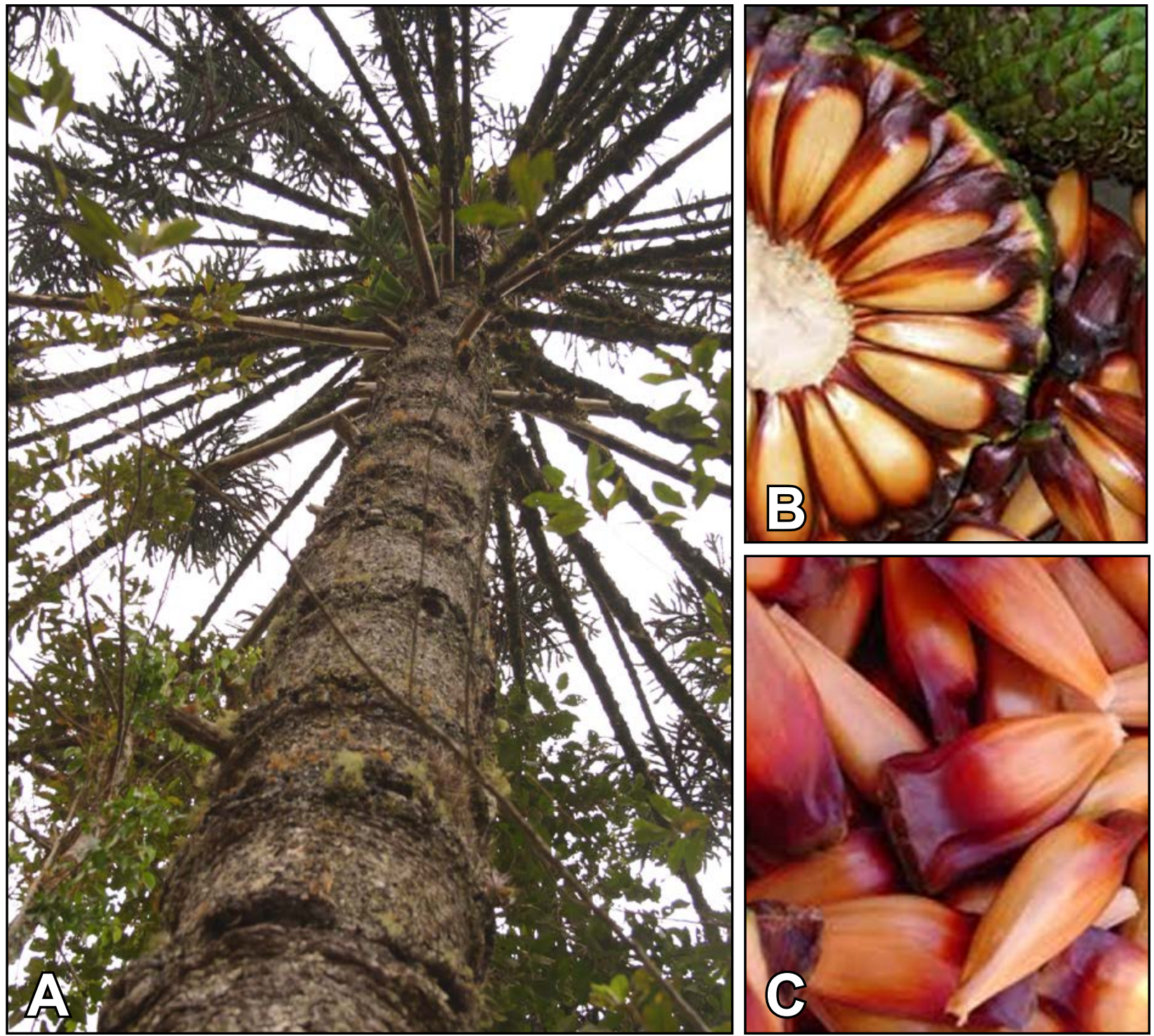

Figure 5. Araucaria angustifolia (Bertol.) Kuntze tree (A), strobilus (B), and seeds (C).

tion of 0 . altimontana may represent a serious risk to the preservation of this species.

The gymnosperm $A$. angustifolia is the symbol of the Paraná Pine Forest. In this study, $A$. angustifolia was the species most mentioned across categories of use. The local importance of this species is evident by the annual local Pine Nut Fair. Although pine nut is a non-timber forest product, Brazilian law regulates its extraction and trade (Brazilian Forestry Code, law 4771 of September $15,1965)$. Pine strobili are freely sold in large quantities, especially the cones (seeds) intended for food and handicrafts (Figure 5). Some families sell large amounts during harvest season in order to increase income, and sales in small scale are also observed in many local markets. As a forest species, cutting $A$. angustifolia is forbidden by I-
BAMA (Aquino 2005). In lieu of this, the local community frequently prevents this species' growth in order to permit space for future buildings or other private property use. This practice, predominantly motivated to improve tourism, results in attitudes incompatible with the aimed preservation of the species. As seen previously, Aquino (2005) pointed out that laws pertaining to the use of species exclusively for species preservation typically only emphasize restrictions and punishments. However, these laws lack recommendations of methods favoring initiatives towards planting for commercial purposes.

\section{Plants used for alimentation}

Silva and Andrade (2005) wrote that a near-equal proportion of arboreal and herbaceous species should indicate 


\section{Quinteiro et al. - Inventory and Implications of Plant Use for Environmental Conservation in Visconde de Mauá, Serra da Mantiqueira, Brazil}

the existence of many fruit ranches in the community; however, Visconde de Mauá showed a predominance of herbaceous species. Other populations living in Atlantic Forest peripheries rarely go to the forest in search of food, yet, when occurring, residents predominantly seek out fruits (Cunha \& Albuquerque 2006). Contradictorily, the traditional fisherman communities along the Atlantic Forest coast more frequently use the edible plants found in their natural habitat, probably because they preserved the way of life of the ancient people (Fonseca-Kruel \& Peixoto 2004, Hanazaki et al. 2000).

\section{Conclusion}

The ethnobotanical knowledge in Visconde de Mauá is the result of the historical conglomeration of several groups, which came into the region with distinct and diverse origins. However, the specific analysis of key informant characteristics shows its similarity to those from ethnobotanical works involving traditional communities. Therefore the group can be considered as a vestige of the local natives or direct descendants of these local indigenous populations. The key informants are linked to the preservation of traditional values and cultures on plants and regional landscapes and can assimilate knowledge from different ethnicities. However, their knowledge has not been substantially transmitted to younger generations, thus making ethnoscientific nature study an urgent matter.

Although the medicinal category of use includes the highest number of useful species, it is unlikely that it represents a threat to local conservation. Specifically, the reported species are cultivated more than they are collected, and the plant parts used, mostly leaves and branches, do not compromise the viability of the individual plants.

Other categories less representative in numeric terms of useful species, such as craftwork, construction, and fuel wood, show a more intense exploitation via use of seeds and entire trees. This is likely to be incompatible with local conservation. In this context, $O$. altimontana and $A$. angustifolia are highlighted as extensively used species, indicating a priority for local conservation management projects.

The low percentage of edible plant crops, the undesirability of searching for such plants in the forest, and the poor commercial offering of such items may result in reduced community use of such species. In this context, tourism is potentially contributing to the decline in horticulture and fruit cultivation and ultimately undermining local traditional culture.

The current environmental policy at Serra da Mantiqueira Environmental Protection Area, in Visconde de Mauá, is dependent on oppression and lacks scientific background and social legitimacy; it could propose more alternatives and educational solutions regarding the use of local plant resources. Ignoring the conservationist potential of the different cultures that live inside this protected area, area managers created policy insufficient for the complete reproduction of the ecosystems, biodiversity conservation, and cultural plurality. Therefore, future policy should include people's perspective from within the conservation area, as well as the investment of their identity recognition. This ultimately will value their knowledge and improve their living conditions as a means to guarantee their participation in the elaboration of a conservation policy that will benefit the protected areas as well.

\section{Acknowledgments}

The authors are grateful to all the informants in the community of Visconde de Mauá that gently agreed to participate in this research; to Mark Hickie for language revision; Haroldo Cavalcante de Lima and José Eduardo Meireles for Ormosia altimontana identification; to Instituto Brasileiro do Meio Ambiente (IBAMA) that authorized this study; and to CNPq and CAPES and PROEX/UFF for the scholarships.

\section{Literature Cited}

Albuquerque, U.P. 1999. La importância de los estúdios etnobiológicos para estabelecimiento de estratégias de manejo y conservación em las florestas tropicales. Biotemas 12:31-47.

Albuquerque, U.P. \& L.H.C. Andrade. 2002. Conhecimento botânico tradicional e conservação em uma área de caatinga no estado de Pernambuco, nordeste do Brasil. Acta Botanica Brasilica 16:273-285. dx.doi.org/10.1590/ $\underline{\text { S0102-33062002000300004 }}$

Albuquerque, U.P. \& R.F.P. Lucena. 2004. Métodos e Técnicas na Pesquisa Etnobotânica. Livro rápido/NUPEEA, Recife, Brasil.

Aquino, F.M. 2005. Cultivo de Araucaria angustifolia: Análise de viabilidade econômico-financeira. Banco Regional de Desenvolvimento do Extremo Sul (BRDE), Florianópolis, Santa Catarina, Brasil.

Arruda, R.S.V. 1997. "Populações tradicionais" e a proteção dos recursos naturais em Unidades de Conservação. Pp. 273-288 in Etnoconservação: Novos rumos para a conservação da natureza nos trópicos. Edited by A.C. Diegues. Núcleo de Apoio à Pesquisa sobre Populações Humanas em Áreas Úmidas Brasileiras, São Paulo, Brasil.

Azevedo, S.K.S. \& I.M. Silva. 2006. Plantas medicinais e de uso religioso comercializadas em mercados e feiras livres no Rio de Janeiro, RJ, Brasil. Acta Botani- 
ca Brasilica 20:185-194. dx.doi.org/10.1590/S0102$\underline{33062006000100017}$

Bailey, K. 1994. Methods of Social Research. $4^{\text {th }}$ edition. The Free Press, New York, New York, U.S.A.

Borba, A.M. \& M. Macedo. 2006. Plantas medicinais usadas para a saúde bucal pela comunidade do bairro Santa Cruz, Chapada dos Guimarães, MT, Brasil. Acta Botanica Brasilica 20(4):771-782. dx.doi.org/10.1590/S0102$\underline{33062006000400003}$

Buckley, R. 2012. Sustainable tourism: Research and reality. Annals of Tourism Research 39(2):528-546. dx.doi. org/10.1016/j.annals.2012.02.003

Câmara, I.G. 2003. Brief history of conservation in the Atlantic forest. Pp. 31-42 in The Atlantic Forest of South America: Biodiversity status, threats, and outlook. Edited by C. Galindo-Leal \& I.G. Câmara. Center for Applied Biodiversity Science \& Island Press, Washington, U.S.A.

Cunha, L.V.F.C. \& U.P. Albuquerque. 2006. Quantitative ethnobotany in an Atlantic Forest fragment of northeastern Brazil: Implications to conservation. Environmental Monitoring and Assessment 114(1-3):1-25. dx.doi. org/10.1007/s10661-006-1074-9

Diegues, A.C. 1996. O Mito Moderno da Natureza Intocada. Hucitec, São Paulo, Brasil.

Di Stasi, L.C. 1996. Plantas Medicinais: Arte e ciência. Um guia de estudo interdisciplinar. Ed. Universidade Estadual Paulista, São Paulo, Brasil.

Fonseca-Kruel, V.S. \& A.L. Peixoto. 2004. Etnobotânica na Reserva Extrativista Marinha de Arraial do Cabo, RJ, Brasil. Acta Botanica Brasilica 18(1):177-190. dx.doi. org/10.1590/S0102-33062004000100015

Fonseca-Kruel, V.S., A.L. Peixoto, C.F.C. Sá, D.S.D. Araújo, W.L. Silva \& A.J. Ferreira. 2006. Plantas Úteis da Restinga: O saber dos pescadores artesanais de Arraial do Cabo. Instituto de Pesquisas Jardim Botânico do Rio de Janeiro, Rio de Janeiro, Brazil.

Hanazaki, N., J.Y. Tamashiro, H.F. Leitão-Filho \& A. Begossi. 2000. Diversity of plant uses in two Caiçara communities from the Atlantic Forest coast, Brazil. Biodiversity and Conservation 9(5):597-615. dx.doi. org/10.1023/A:1008920301824

IBAMA. 2007. Instituto Brasileiro de Meio Ambiente. www. ibama.gov.br Accessed 8 September 2007.

Kramer, K.U. \& P.S. Green. 1990. The Families and Genera of Vascular Plants, Volume 1. Pteridophytes and
Gymnosperms. Edited by K. Kubitzki. Springer-Verlag, New York, New York, U.S.A.

Lista de Espécies da Flora do Brasil. 2014. Jardim Botânico do Rio de Janeiro. http://floradobrasil.jbri.gov.br/ Accessed 31 August 2014.

Medeiros, M.F.T., V.S. Fonseca \& R.H.P. Andreata. 2004. Plantas medicinais e seus usos pelos sitiantes da Reserva Rio das Pedras, Mangaratiba, RJ, Brasil. Acta Botanica Brasilica 18(2):391-399. dx.doi.org/10.1590/S0102$\underline{33062004000200019}$

Meireles, J.E. 2014. Ormosia. In Lista de Espécies da Flora do Brasil. Jardim Botânico do Rio de Janeiro. http:// reflora.jbri.gov.br/jabot/floradobrasil/FB134140 Accessed 31 August 2014.

Myers, N., R.A. Mittermeier, C.G. Mittermeier, G.A.B. Fonseca \& J. Kent. 2000. Biodiversity hotspots for conservation priorities. Nature 403:853-858. dx.doi. org/10.1038/35002501

Neves, E.M.S.C. \& S.W. Maia. 2012. Governança ambiental e cooperação intergovernamental no Brasil: Lições de Visconde de Mauá. Revista Iberoamericana de Economía Ecológica 18:21-35. http://redibec.org/IVO/rev18 02.pdf

Oliveira-Filho, A.T., D.A. Carvalho, M.A.L. Fontes, E. Van Den Berg, N. Curi \& W.A.C. Carvalho. 2004. Variações estruturais do compartimento arbóreo de uma floresta semidecídua alto-montana na chapada das Perdizes, Carrancas, MG. Revista Brasileira de Botânica 27:291-309.

Pasa, M.C., J.J. Soares \& G. Guarim-Neto. 2005. Estudo etnobotânico na comunidade de Conceição-Açu (alto da bacia do rio Aricá Açu, MT, Brasil). Acta Botanica Brasilica 19(2):195-207. dx.doi.org/10.1590/S010233062005000200001

Pavan-Fruehauf, S. 2000. Plantas Medicinais de Mata Atlântica: Manejo sustentado e amostragem. Annablume/ Fapesp, São Paulo, Brasil.

Pereira, I.M., A.T. Oliveira-Filho, S.A. Botelho, W.A.C Carvalho, M.A.L. Fontes, I. Schiavini \& A.F. Silva. 2006. Composição florística do compartimento arbóreo de cinco remanescentes florestais do maciço do Itatiaia, Minas Gerais e Rio de Janeiro. Rodriguesia 57:103-126.

Pinto, E.P.P., M.C.M. Amorozo \& A. Furlan. 2006. Conhecimento popular sobre plantas medicinais em comunidades rurais Mata Atlântica - Itacaré, BA, Brasil. Acta Botanica Brasilica 20(4):751-762. dx.doi.org/10.1590/ S0102-33062006000400001 


\section{Quinteiro et al. - Inventory and Implications of Plant Use for Environmental Conservation in Visconde de Mauá, Serra da Mantiqueira, Brazil}

Reveal, J.L. \& M.W. Chase. 2011. APG III: Bibliographical information and synonymy of Magnoliidae. Phytotaxa 19:71-134.

Rezende H.A. \& M.I.M. Cocco. 2002. A utilização de fitoterapia no cotidiano de uma população rural. Revista da Escola de Enfermagem USP 36(3):282-288. dx.doi. org/10.1590/S0080-62342002000300011

Ribeiro, M.C., J.P. Metzger, A.C. Martensen, F.J. Ponzoni \& M.M. Hirota. 2009. The Brazilian Atlantic Forest: How much is left and how is the remaining forest distributed? Implications for conservation. Biological Conservation 142(6):1141-1153. dx.doi.org/10.1016/j.biocon.2009.02.021

Richter, M. \& E.M.F.R. Souza. 2013. Avaliação de impactos ecológicos e sociais do uso público no Parque Nacional do Itatiaia - Trilha Alto dos Brejos. Boletim de Geografia 31(1):91-100.

Ruschmann, D.V.M. 2013. Impactos ambientais do turismo ecológico no Brasil. Revista Turismo Em Análise 4(1):5668. $\underline{\text { dx.doi.org/10.11606/issn.1984-4867.v4i1p56-68 }}$

Santos, M.G., P.C.A. Fevereiro, G.L. Reis, J.I. Barcelos \& F.M.M.A. Ney. 2009. Plantas da Restinga: Potencial econômico. Technical Books, Rio de Janeiro, Brasil.

Shanley, P. \& N.A. Rosa. 2005. Conhecimento em erosão: Um inventário etnobotânico na fronteira de exploração da Amazônia oriental. Boletim do Museu Paraense Emílio Goeldi/ Série Ciências Naturais 1:147-171.

Sloan, S., C.N. Jenkins, L.N. Joppa, D.L.A. Gaveau \& W.F. Laurance. 2014. Remaining natural vegetation in the global biodiversity hotspots. Biological Conservation 177:12-24. dx.doi.org/10.1016/j.biocon.2014.05.027

Schardong, R.M.F. \& A.C. Cervi. 2000. Estudo etnobotânico de plantas medicinais e místico na comunidade de São Benedito, Bairro São Francisco, Campo Grande, MS. Acta Biologica Paranaense 29:187-217.

Silva, A.J.R. \& L.H.C. Andrade. 2005. Etnobotânica nordestina: Estudo comparativo da relação entre comunidades e vegetação na Zona do Litoral - Mata do Estado de Pernambuco, Brasil. Acta Botanica Brasilica 19(1):4560. dx.doi.org/10.1590/S0102-33062005000100006

Smith, A.R., K.M. Pryer, E. Schuettpelz, P. Korall, H. Schneider \& P.G. Wolf. 2006. A classsification for extant ferns. Taxon 55(3):705-731. dx.doi.org/10.2307/25065646

Vendruscolo, G.S. \& L.A. Mentz. 2006. Estudo da concordância das citações de uso e importância das espécies e famílias utilizadas como medicinais pela comunidade do bairro Ponta Grossa, Porto Alegre, RS, Brasil. Acta Botanica Brasilica 20(2):367-382. dx.doi.org/10.1590/ S0102-33062006000200012

Viana, V.M. \& A.J.A. Tabanez. 1996. Biology and conservation of forest fragments in Brazilian Atlantic moist forest. Pp. 151-167 in Forest Patches in Tropical Landscapes. Edited by J. Schellas \& R. Greenberg. Island Press, Washington, U.S.A.

Voeks, R.A. 2007. Are women reservoirs of traditional plant knowledge? Gender, ethnobotany and globalization in northeast Brazil. Singapore Journal of Tropical Geography 28(1):7-20. dx.doi.org/10.1111/j.14679493.2006.00273.x

Voeks, R.A. \& A. Leony. 2004. Forgetting the forest: Assessing medicinal plant erosion in eastern Brazil. Economic Botany 58(1):294-306. dx.doi.org/10.1663/00130001(2004)58[S294:FTFAMP]2.0.CO;2

Zamith, L.R. \& F.R. Scarano. 2004. Produção de mudas de espécies das Restingas do município do Rio de Janeiro, RJ, Brasil. Acta Botanica Brasilica 18(1):161-176. dx.doi.org/10.1590/S0102-33062004000100014 
